[Article]

\title{
$p-\pi$ 共轭分子的氨基面外弯曲振动模的拉曼光谱
}

\author{
陶 莎 于利娟吴德印 ${ }^{*}$ 田中群 \\ (厦门大学化学化工学院化学系, 固体表面物理化学国家重点实验室, 福建厦门 361005)
}

\begin{abstract}
摘要：拉曼光谱是一种用途广泛的无损分子检测技术, 其能够提供化学物质的分子结构指纹信息. 一种面外 弯曲振动模被称作 wagging 振动, 它的信号尤为特殊, 其频率和强度都非常依赖于检测环境. 以乙烯胺和苯胺 为例, 采用密度泛函理论计算研究了 $p-\Pi$ 共轭分子分别与水簇和银簇作用的平衡结构、成键作用和拉曼光谱. 结果表明, 弱相互作用, 如分子与金属表面的弱吸附作用以及分子与水之间的氢键作用, 均使氨基面外弯曲振 动模 $\left(\omega_{\mathrm{NH}_{2}}\right)$ 的拉曼信号发生显著的变化. 考虑溶剂化效应后, 氢键作用减弱, 计算拉曼光谱趋于一致. 通过进一 步对电子结构的分析, 解释了面外弯曲振动信号显著增强的原因, 揭示了面外弯曲振动模与分子 $p-\pi$ 共轭作用 之间的关系.
\end{abstract}

关键词：面外弯曲振动模；分子间弱相互作用；氢键； 乙烯胺；苯胺；拉曼光谱；密度泛函理论 中图分类号: 0641

\section{Raman Spectra of Amino Wagging Vibrational Modes in $p-\pi$-Conjugated Molecules}

\author{
TAO Sha YU Li-Juan WU De-Yin* TIAN Zhong-Qun \\ (State Key Laboratory for Physical Chemistry of Solid Surfaces, Department of Chemistry, College of Chemistry and \\ Chemical Engineering, Xiamen University, Xiamen 361005, Fujian Province, P. R. China)
}

\begin{abstract}
Raman spectroscopy has been widely used as a non-destructive testing and molecular recognition technology, providing fingerprint information for chemical and biological molecular structures. One type of out-of-plane bending vibration observed in Raman spectroscopy is named the 'wagging vibration'. The Raman signal of the wagging mode is very sensitive; not only the vibrational frequency but also the Raman intensity depends strongly on environment factors. In this report, density functional theory (DFT) calculations are used to study the equilibrium structures, binding interactions, and Raman spectra of vinylamine and aniline as well as their complexes with silver clusters and water clusters. Vinylamine-silver and aniline-silver clusters were used to simulate the interactions of the molecules adsorbed on silver surfaces, while vinylamine-water and aniline-water clusters were used to investigate the hydrogen bonding interactions of vinylamine and aniline with water clusters. Our calculated results show that the Raman signal of the amino wagging mode strongly depends on the hydrogen bonding interaction of the nitrogen lone pair in the amino group with the $\mathrm{O}-\mathrm{H}$ bond of water. Increasing the size of the water clusters causes a large blue shift and considerable enhancement in the intensity of the wagging vibration. When the polarized continuum model was used to consider the solvation effect, the electrostatic interaction contributing to the hydrogen bond was weakened. In this case, the simulated Raman spectra were similar to each other. For vinylamine and aniline interacting with silver clusters, the Raman signals of the amino wagging vibration were changed by the weak binding interaction, revealing the relationship between the abnormal signal of wagging vibrations and the weak interaction in $p-\pi$-conjugated systems.
\end{abstract}

Received: April 16, 2013; Revised: May 31, 2013; Published on Web: June 3, 2013.

"Corresponding author. Email: dywu@xmu.edu.cn; Tel: +86-592-2189023.

The project was supported by the National Natural Science Foundation of China (91027009, 21021002, 20973143), National Key Basic Research Program of China (973) (2009CB930703), and Xiamen University, China (2010121020).

国家自然科学基金(91027009, 21021002, 20973143), 国家重点基础研究发展规划项目(973) (2009CB930703)和厦门大学(2010121020)资助

(C) Editorial office of Acta Physico-Chimica Sinica 
Key Words: Wagging vibration mode; Weak intermolecular interaction; Hydrogen bond; Vinylamine; Aniline; Raman spectroscopy; Density functional theory

\section{1 引言}

弱相互作用是一类普遍存在的作用, 其中氢键 作用占有重要的地位. 在生物分子体系中, 含有芳 香氨基基团的许多分子与其环境形成氢键作用, 但 这类研究体系往往较为复杂, 难于进行系统研究. 近些年来, 苯胺分子已经成为一个很好的模型分 子. ${ }^{1-5}$ 前人已对苯胺分子与其它小分子的红外光谱 进行测量, 通过分析氨基伸缩振动谱峰的变化探讨 分子间氢键作用. 如 Nakanaga等 ${ }^{6-9}$ 用红外光谱研究 了苯胺与一系列小分子 (如 $\mathrm{H}_{2} \mathrm{O} 、 \mathrm{NH}_{3} 、 \mathrm{~N}_{2}$ 和 $\mathrm{CO}$ 等)之 间的氢键作用, 发现苯胺分子的氨基与小分子的氮 或氧原子形成氢键, 其导致氨基的 $\mathrm{N}-\mathrm{H}$ 伸缩振动 发生几十个波数的红移. 然而, 人们目前普遍关注 的是这种弱相互作用对高频区谱峰的影响, 很少有 人关注氢键作用对氨基的面外弯曲振动模谱峰 $\left(\omega_{\mathrm{NH}_{2}}\right)$ 的影响. 实际上氨基上氮与氢给体形成的氢键 作用更强, ${ }^{10,11}$ 其引起频率的位移更大, 谱峰强度变 化也更显著.

拉曼光谱是一种非弹性散射光谱, 它以单色性 很好的激光作为光源, 能够提供化学和生物分子结 构的指纹信息, 广泛用于无损检测和分子识别技 术. 在采用拉曼光谱研究苯胺及苯胺类衍生物分子 与银表面作用的实验中, 氨基面外弯曲振动模的信 号相对于分子发生显著的变化. ${ }^{12-14}$ 当苯胺分子吸附 在银表面上, 人们常在 900-1000 $\mathrm{cm}^{-1}$ 区间观测一个 很强且很宽的拉曼谱峰, 该峰被认为来自于苯环的 呼吸振动或氨基的摇摆振动. ${ }^{15-17}$ 最近, Zhao 等 ${ }^{18}$ 用 密度泛函理论计算了苯胺分子吸附在币族金属表 面的拉曼谱峰, 观察到了 $\omega_{\mathrm{NH}_{2}}$ 振动模的频率明显蓝 移, 同时其拉曼信号显著增强. 他们认为 $\omega_{\mathrm{NH}_{2}}$ 振动频 率的蓝移是由于沿着该振动模的势能曲线由双势 阱转化单势阱, 同时势能曲线的曲率增大. 该振动 模的拉曼强度显著增强可以解释为伴随着 $\omega_{\mathrm{NH}_{2}}$ 振动 氮原子的杂化在 $s p^{2}$ 和 $s p^{3}$ 之间变化, 导致电子密度 的重新分布, 从而极化率导数显著增大.

在本文中, 我们选择了乙烯胺和苯胺分子作为 研究对象, 用密度泛函理论分析这两个分子与水以 及与金属表面作用对分子内 $p-\pi$ 共轭效应及拉曼光 谱的影响, 研究分子间的弱相互作用与氨基面外弯
曲振动的关系, 进一步解释氨基的面外弯曲振动拉 曼信号异常产生的原因.

\section{2 理论计算方法和模型}

为了研究弱相互作用对氨基的面外弯曲振动 模的影响, 我们构造了分子与水簇作用以及分子与 银簇作用的两种理论模型. 选择乙烯胺分子作为探 针分子, 主要是该分子涉及分子内 $p-\pi$ 作用, 且前人 对其分子本身的振动光谱有详细研究. ${ }^{19-21}$ 首先, 对 于乙烯胺和苯胺分子与水作用, 如图 1 所示, 我们主 要考虑水以 $\mathrm{O}-\mathrm{H}$ 键与氨基氮原子形成氢键. 随水 分子数增加, 氨基的 $\mathrm{N}-\mathrm{H}$ 键也与水分子的氧成氢 键作用. 因氢键作用很依赖于介质环境, 我们用溶 质密度(SMD)的溶剂化模型, ${ }^{22}$ 其考虑氢原子的影响 和非静电作用项, 能很好地预测溶剂化的吉布斯自 由能. 我们选择水作为溶剂, 取水体相的相对介电 常数值为 78.3 .

其次, 为了研究分子在银纳米结构表面的吸 附, 选取银簇模拟金属表面的吸附活性位, 研究了 两个分子与银簇的作用对氨基的面外弯曲振动模 的影响. 在分子-银族模型中, 分子与银簇的作用主 要是通过氨基孤对轨道形成配位作用. 因为分子-银 簇的相互作用依赖于吸附位和金属族的大小, 因此 我们选择了不同大小的银簇 $\operatorname{Ag}_{n}(n=1,2,3,4)$ 模拟 银表面可能的活性位. 依据成键规则, 如能量相近、 轨道对称性的匹配和最大重叠, 选择的银簇不仅可 以反映表面活性位的电子结构特性, 而且也能较好 地描述乙烯胺和苯胺与银簇作用的成键作用. 在以 前研究中, 该模型合理地模拟了吡啶分子吸附在粗 䊁金属表面的拉曼光谱. ${ }^{23}$

为了考查不同密度泛函对计算结果的影响, 采 用不同的杂化密度泛函 B3LYP、X3LYP 和 M06进 行了理论计算. 本文所有密度泛函理论计算由 Gaussian 09 程序 ${ }^{24}$ 得到. 在计算中对分子中的原子 采用全电子基组 6-311+ $\mathrm{G}(d, p)$, 其为 $\mathrm{C} 、 \mathrm{~N} 、 \mathrm{O}$ 和 $\mathrm{H}$ 原 子提供了极化函数, 为 $\mathrm{C} 、 \mathrm{~N}$ 和 $\mathrm{O}$ 原子提供了弥散函 数. ${ }^{25,26}$ 对银原子采用相对论噟势和相应基组 LANL2DZ 描述金属内壳层的电子和价层电子. ${ }^{27,28}$ 基于上述方法和基组, 我们计算了苯胺和乙烯胺分 


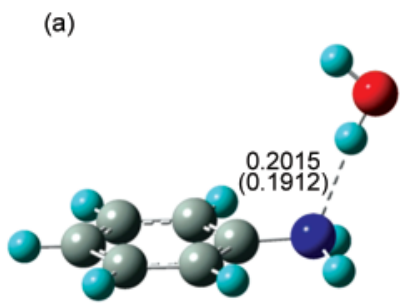

(b)
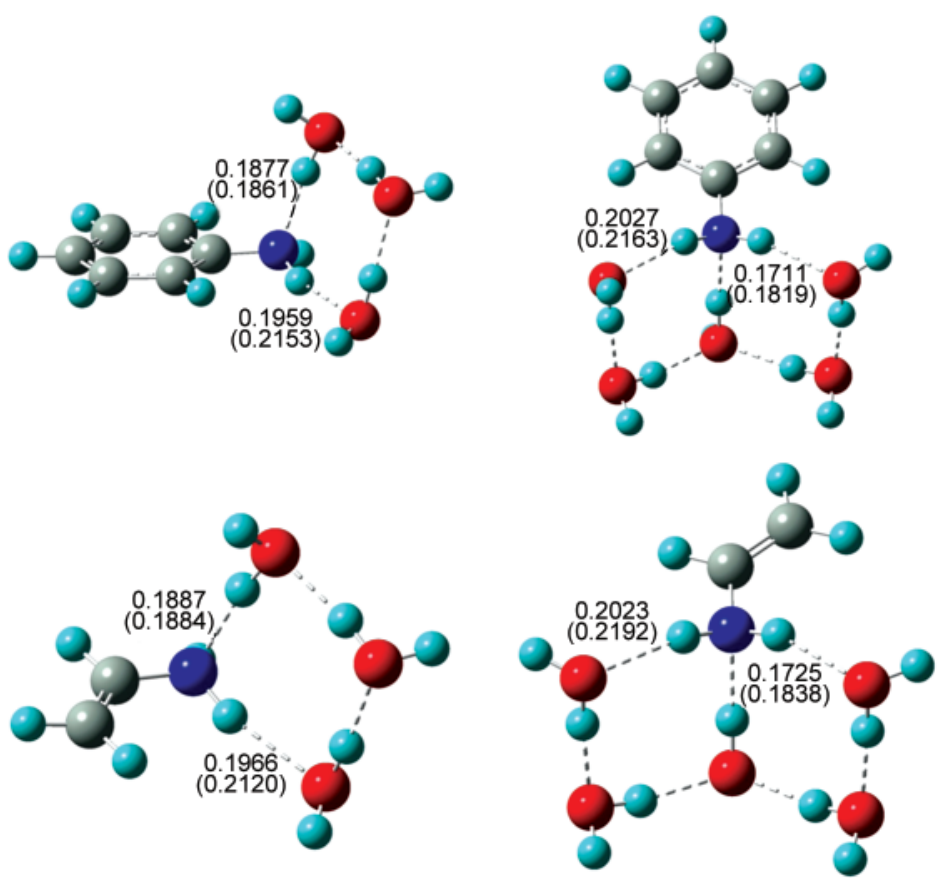

图 1 在 B3LYP/6-311+G( $d, p)$ 水平下计算的气相中苯胺(a)和乙烯胺(b)分子分别与一个、三个和五个水分子的 水簇作用的优化结构

Fig.1 Optimized structures of aniline (a) and vinylamine (b) interacting with one, three, and five water molecules in the gas phase calculated at the B3LYP/6-311+G( $d, p)$ level

The unit of bond length is in $\mathrm{nm}$ and the values in parentheses are calculated by the solute model of density (SMD).

子本身的面外弯曲振动的频率和拉曼散射因子. 同 时, 我们也用 B 3 LYP 方法计算分子-水簇以及分子银簇的成键能和拉曼光谱. 计算分子与金属之间的 成键能定义为: $\mathrm{BE}=-\left(E_{\mathrm{MOL}-\mathrm{M}_{n}}-E_{\mathrm{MOL}}-E_{\mathrm{M}_{n}}\right)$, 其中 $n$ 代表 银簇中银原子的个数, $E_{\mathrm{MOL}-\mathrm{M}_{n}}$ 代表复合物的能量, $E_{\mathrm{MOL}}$ 和 $E_{\mathrm{M}_{n}}$ 分别代表分子和银簇的能量. 在成键能计 算中,也用基组重叠误差(BSSE)校正方法 ${ }^{29,30}$ 来校正 成键能. 还利用自然成键轨道理论 $(\mathrm{NBO})^{31,32}$ 分析分 子间的轨道作用能.

采用公式(1)计算了不同振动模的微分拉曼散 射截面, 其对应于单个分子第 $i$ 个振动模的拉曼强 度, ${ }^{33}$

$$
\left(\frac{\mathrm{d} \sigma}{\mathrm{d} \Omega}\right)_{i}=\frac{(2 \pi)^{4}}{45} \cdot \frac{h}{8 \pi^{2} c \widetilde{v}_{i}} \cdot \frac{\left(\widetilde{v}_{0}-\widetilde{v}_{i}\right)^{4}}{1-\exp \left(-h c \widetilde{v}_{i} / k_{\mathrm{B}} T\right)} S_{i}
$$

其中, $S_{i}=45\left(\frac{\mathrm{d} \sigma}{\mathrm{d} Q_{i}}\right)^{2}+7\left(\frac{\mathrm{d} \gamma}{\mathrm{d} Q_{i}}\right)^{2}$

式中, $h 、 c 、 k_{\mathrm{B}}$ 和 $T$ 分别为 Planck 常数、光速、 Boltzmann 常数和 Kelvin 温度. $\widetilde{v}_{0}$ 和 $\widetilde{v}_{i}$ 分别为激发 光频率 $\left(\mathrm{cm}^{-1}\right)$ 和第 $i$ 个振动模的振动频率 $\left(\mathrm{cm}^{-1}\right)$. 为 了与实验拉曼谱进行比较, 我们也对 200-1800 $\mathrm{cm}^{-1}$ 波数的密度泛函计算谐频率采用 0.981 进行校正. $S_{i}$
为拉曼散射因子. $\mathrm{d} \alpha / \mathrm{d} Q_{i}$ 和 $\mathrm{d} \gamma / \mathrm{d} Q_{i}$ 分别为极化率的各 向同性部分和各向异性部分对第 $i$ 个简正坐标的导 数. 在理论计算拉曼光谱中, 对计算微分拉曼散射 截面进行 Lorentzian 展开, 采用最大高度半高宽为 $10 \mathrm{~cm}^{-1}$, 激发光波长为 $514.5 \mathrm{~nm}$.

\section{3 计算结果与讨论}

\section{1 苯胺和乙烯胺分子的拉曼光谱}

我们利用不同的杂化密度泛函 B3LYP、X3LYP 和 M06 的理论计算方法和 6-311+ $\mathrm{G}(d, p)$ 基组计算 了甲胺、乙烯胺和苯胺分子的面外弯曲振动的频率 和强度, 比较不同烃基与氨基相连时面外弯曲振动 谱性质的变化. 表 1 给出三种不同理论方法计算的 面外弯曲振动模的频率和拉曼散射因子. 结果表明 计算方法对结果影响不大, 与甲胺的 $\omega_{\mathrm{NH}_{2}}$ 频率相比, 乙烯胺和苯胺的频率明显减小, 并且这两者较接 近. 这主要是由于在甲胺分子中氨基是 $s p^{3}$ 杂化, 而 在乙烯胺和苯胺分子内存在 $p-\pi$ 共轭效应, 氨基上 的孤对与乙烯基或苯环共轭导致其倾向于 $s p^{2}$ 杂化 性质. 由此可见氨基面外弯曲振动模的频率与氨基 的杂化性质紧密相关. 在氨分子中, 其伞式振动频 
表 1 用不同密度泛函理论方法计算的甲胺、乙烯胺和苯胺的氨基面外弯曲振动的拉曼频率 $\left(\omega_{i}\right)$ 和拉曼强度 $\left(S_{i}\right)$

Table 1 Vibrational Raman frequencies $\left(\omega_{i}\right)$ and intensities $\left(S_{i}\right)$ of $\omega_{\mathrm{NH}_{2}}$ calculated by different density functional theory methods for methylamine, vinylamine, and aniline

\begin{tabular}{|c|c|c|c|c|c|c|c|}
\hline \multirow{2}{*}{ Species } & \multicolumn{2}{|r|}{ B3LYP } & \multicolumn{2}{|r|}{ X3LYP } & \multicolumn{2}{|r|}{ M06 } & \multirow{2}{*}{$\begin{array}{c}\text { Expt. } \\
\omega_{i} / \mathrm{cm}^{-1}\end{array}$} \\
\hline & $\omega_{i} / \mathrm{cm}^{-1}$ & $10^{4} S_{i} /\left(\mathrm{nm}^{4} \cdot \mathrm{amu}^{-1}\right)$ & $\omega_{i} / \mathrm{cm}^{-1}$ & $10^{4} S_{i} /\left(\mathrm{nm}^{4} \cdot \mathrm{amu}^{-1}\right)$ & $\omega_{i} / \mathrm{cm}^{-1}$ & $10^{4} S_{i} /\left(\mathrm{nm}^{4} \cdot \mathrm{amu}^{-1}\right)$ & \\
\hline $\mathrm{CH}_{3} \mathrm{NH}_{2}$ & 808 & 2.98 & 808 & 2.93 & 801 & 2.12 & $780^{\mathrm{a}}$ \\
\hline $\mathrm{C}_{2} \mathrm{H}_{3} \mathrm{NH}_{2}$ & 544 & 3.86 & 546 & 1.84 & 534 & 1.24 & $570^{\mathrm{b}}$ \\
\hline $\mathrm{C}_{6} \mathrm{H}_{5} \mathrm{NH}_{2}$ & 564 & 6.88 & 562 & 6.91 & 544 & 7.61 & $660^{\mathrm{c}}$ \\
\hline
\end{tabular}

${ }^{\mathrm{a}}$ from Ref. $35 ;{ }^{\mathrm{b}}$ from Ref. $19 ;{ }^{\mathrm{c}}$ from Ref. 36

率是 $958 \mathrm{~cm}^{-1} .34$ 甲基取代氨的一个氢后, 三种泛函 预测甲胺的 $\omega_{\mathrm{NH}_{2}}$ 频率为 808 和 $801 \mathrm{~cm}^{-1}$, 其略大于实 验观测值 $\left.\left(780 \mathrm{~cm}^{-1}\right)\right)^{35}$ 在乙烯胺和苯胺中, 由于 $p-\pi$ 共轭作用, 理论计算谐振动频率小于甲胺中相应的 频率, 而实验观测的频率分别为 570 和 $660 \mathrm{~cm}^{-1}, 19,36$ 其大于理论计算谐振动频率. 这主要是观测频率不 是振动基频, 而是由于该振动模均具有显著的非谐 性, 在自由分子中具有双极小势阱, 因此观测频率 为泛频. ${ }^{37}$ Rauhut 和 Pulay ${ }^{38}$ 建议其谐振动频率为 541 $\mathrm{cm}^{-1}$, 与表 1 计算频率接近.

图 2 是采用密度泛函理论计算乙烯胺和苯胺分 子内涉及 $p-\pi$ 共轭作用的轨道图. 在氨基与乙烯基 和苯基形成 $\mathrm{C}-\mathrm{N} \sigma$ 键的同时, 氨基的孤对轨道也与 $\pi$ 轨道发生作用. 在乙烯胺分子中, 我们发现氨基的 孤对轨道主要与乙烯基的 $\pi$ 成键轨道作用, 形成乙 烯胺分子中的一个成键轨道和一个反键轨道. 在苯

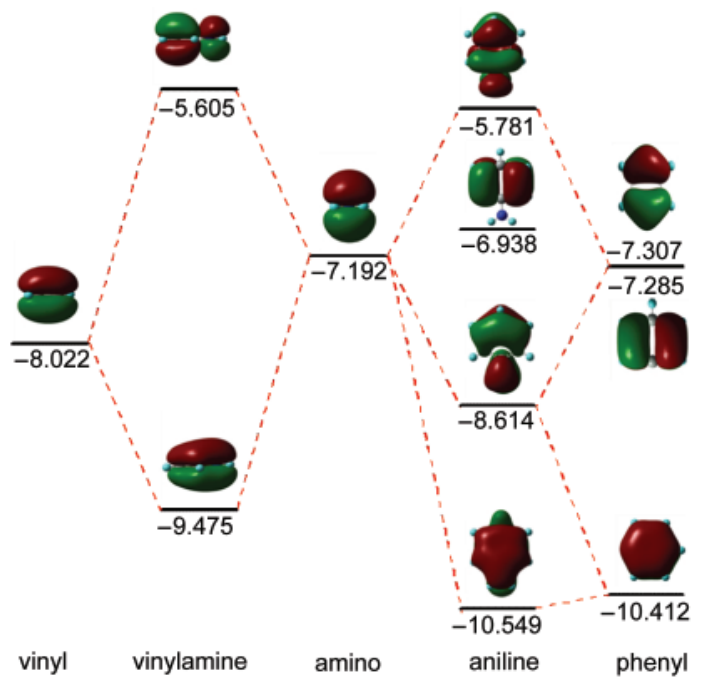

图 2 氨基的孤对轨道分别与乙烯基和苯基的 $\pi$ 轨道作用 形成乙烯胺和苯胺的 $p-\pi$ 共轭轨道的轨道能级图

Fig.2 Plots of $p-\pi$ conjugated orbitals in vinylamine and aniline formed from the lone pair orbital in the amino group interacting with the $\pi$ orbitals in vinyl and phenyl groups
胺分子中, 氨基的孤对与苯基的两个 $\pi$ 成键轨道相 互作用, 形成苯胺分子中三个与 $p-\pi$ 共轭作用相关 的分子轨道. 在这两个分子中, 上面的分析表明轨 道作用主要发生在占据轨道之间, 未占据的 $\pi$ 反键 轨道不产生明显影响.

\section{2 氢键作用对氨基面外弯曲振动模的影响}

图 1 中也给出苯胺和乙烯胺分子以氨基与不同 大小的水簇作用的优化结构, 它们将用于研究氢键 作用对分子中氨基面外弯曲振动模的拉曼信号的 影响. 图中标出了与分子间氢键相关的 $\mathrm{N} \cdots \mathrm{H}-\mathrm{O}$ 和 $\mathrm{O} \cdots \mathrm{H}-\mathrm{N}$ 的氢键键长, 括号中为采用溶剂化 SMD 模型计算的结构参数. 比较 $\mathrm{N} \cdot \cdot \mathrm{H}-\mathrm{O}$ 氢键结构的变 化可以看出, 在苯胺和乙烯胺中随着水分子数目的 增加, $\mathrm{N} \cdots \mathrm{H}$ 的距离均逐渐变短. 用 $\mathrm{NBO}$ 分析计算氨 基氮孤对与 $\mathrm{O}-\mathrm{H}$ 键反键轨道作用能, 得到在苯胺 复合物中其分别为 $18.07 、 32.72$ 和 $64.02 \mathrm{~kJ} \cdot \mathrm{mol}^{-1}$, 而 在乙烯胺复合物中其依次为 $17.24 、 31.46$ 和 60.96 $\mathrm{kJ} \cdot \mathrm{mol}^{-1}$. 表明 $\mathrm{N} \cdots \mathrm{H}-\mathrm{O}$ 氢键随水分子数目增大该 氢键作用增强. 这也与表 2 计算的分子之间的氢键 的键能越来越大相一致. 含有三个水分子和五个水 分子的氢键复合物, 其 $\mathrm{N}-\mathrm{H} \cdots \mathrm{O}$ 氢键的 $\mathrm{H} \cdots \mathrm{O}$ 原子 距离随水分子数增加而增长. 这说明氨基的氮原子 与水分子的氢原子之间的氢键作用随水分子数增 加会变得更加稳定.

图 3 是在 B3LYP/6-311+G $(d, p)$ 水平上计算分子水簇的拉曼光谱. 图中标出了含有氨基面外弯曲振 动贡献的谱峰, 其中用*标出的为亚甲基面外弯曲 振动的谱峰. 图 3(A, C) 是在气相中计算的拉曼光 谱. 从图上可以看到, 不论是苯胺还是乙烯胺, 其氨 基的面外弯曲振动模均依赖于氨基周围水分子的 数目, 与其分子本身的拉曼光谱(表 1)相比, 频率有 明显的蓝移, 强度有明显的增大. 如图 3(A)所示, 当 苯胺与不同水簇作用时, 在 $700-1000 \mathrm{~cm}^{-1}$ 的波数范 围内均出现三个包含 $\omega_{\mathrm{NH}_{2}}$ 振动坐标的谱峰. 通过对 振动谱峰的分析, 表 2 结果表明它们来自于面外弯 
表 2 在气相和溶剂化下理论计算苯胺、乙烯胺与水簇复合物的包含面外弯曲振动模的频率 $\left(\omega_{i}\right)$ 、拉曼强度 $\left(S_{i}\right)$ 、

氢键键能(BE)以及振动模的归属

Table 2 Calculated frequencies $\left(\omega_{i}\right)$, Raman activity $\left(S_{i}\right)$, hydrogen bonding energy $(\mathrm{BE})$, and vibrational assignment of $\omega_{\mathrm{NH}_{2}}$ for $\mathrm{C}_{6} \mathrm{H}_{5} \mathrm{NH}_{2}-n \mathrm{H}_{2} \mathrm{O}$ and $\mathrm{C}_{2} \mathrm{H}_{3} \mathrm{NH}_{2}-n \mathrm{H}_{2} \mathrm{O}$ complexes $(n=1,3,5)$ in the gas phase as well as that with SMD model

\begin{tabular}{|c|c|c|c|c|}
\hline Species & $\omega_{i} / \mathrm{cm}^{-1}$ & $10^{4} S_{i} /\left(\mathrm{nm}^{4} \cdot \mathrm{amu}^{-1}\right)$ & Assignment & $\mathrm{BE} /\left(\mathrm{kJ} \cdot \mathrm{mol}^{-1}\right)$ \\
\hline \multirow[t]{3}{*}{$\mathrm{C}_{6} \mathrm{H}_{5} \mathrm{NH}_{2}-\mathrm{H}_{2} \mathrm{O}$} & 817 & 23.94 & $\omega_{\mathrm{NH}_{2}}$, ring breathing & 23.35 \\
\hline & 771 & 5.70 & $\omega_{\mathrm{NH}_{2}}, \gamma_{\mathrm{CH}}$ & \\
\hline & 727 & 6.15 & $\omega_{\mathrm{NH}_{2}}, \gamma_{\mathrm{CH}}$ & \\
\hline \multirow[t]{3}{*}{$\mathrm{C}_{6} \mathrm{H}_{5} \mathrm{NH}_{2}-3 \mathrm{H}_{2} \mathrm{O}$} & 946 & 19.50 & $\omega_{\mathrm{NH}_{2}}$ & 43.01 \\
\hline & 890 & 3.87 & $\omega_{\mathrm{NH}_{2}}, \gamma_{\mathrm{CH}}$ & \\
\hline & 868 & 15.22 & $\omega_{\mathrm{NH}_{2}}, \gamma_{\mathrm{CH}}$ & \\
\hline \multirow[t]{3}{*}{$\mathrm{C}_{6} \mathrm{H}_{5} \mathrm{NH}_{2}-5 \mathrm{H}_{2} \mathrm{O}$} & 1004 & 4.51 & $\omega_{\mathrm{NH}_{2}}$ & 44.39 \\
\hline & 982 & 63.14 & $\omega_{\mathrm{NH}_{2}}, \gamma_{\mathrm{CH}}$ & \\
\hline & 974 & 11.07 & $\omega_{\mathrm{NH}_{2}}, \gamma_{\mathrm{CH}}$ & \\
\hline \multirow[t]{3}{*}{$\mathrm{C}_{6} \mathrm{H}_{5} \mathrm{NH}_{2}-\mathrm{H}_{2} \mathrm{O}-\mathrm{SMD}$} & 819 & 85.82 & $\omega_{\mathrm{NH}_{2}}$, ring breathing & 14.27 \\
\hline & 769 & 32.11 & $\omega_{\mathrm{NH}_{2}}, \gamma_{\mathrm{CH}}$ & \\
\hline & 723 & 41.31 & $\omega_{\mathrm{NH}_{2}}, \gamma_{\mathrm{CH}}$ & \\
\hline \multirow[t]{3}{*}{$\mathrm{C}_{6} \mathrm{H}_{5} \mathrm{NH}_{2}-3 \mathrm{H}_{2} \mathrm{O}-\mathrm{SMD}$} & 818 & 77.31 & $\omega_{\mathrm{NH}_{2}}$, ring breathing & 35.31 \\
\hline & 771 & 27.81 & $\omega_{\mathrm{NH}_{2}}, \gamma_{\mathrm{CH}}$ & \\
\hline & 740 & 22.79 & $\omega_{\mathrm{NH}_{2}}, \gamma_{\mathrm{CH}}$ & \\
\hline \multirow[t]{3}{*}{$\mathrm{C}_{6} \mathrm{H}_{5} \mathrm{NH}_{2}-5 \mathrm{H}_{2} \mathrm{O}-\mathrm{SMD}$} & 840 & 152.77 & $\omega_{\mathrm{NH}_{2}}$, ring breathing & 25.40 \\
\hline & 794 & 34.10 & $\omega_{\mathrm{NH}_{2}}$, ring breathing & \\
\hline & 751 & 15.91 & $\omega_{\mathrm{NH}_{2}}, \gamma_{\mathrm{CH}}$ & \\
\hline \multirow[t]{2}{*}{$\mathrm{C}_{2} \mathrm{H}_{3} \mathrm{NH}_{2}-\mathrm{H}_{2} \mathrm{O}$} & 838 & 8.18 & $\omega_{\mathrm{CH}_{2}}$ & 22.43 \\
\hline & 780 & 2.58 & $\omega_{\mathrm{NH}_{2}}$ & \\
\hline \multirow[t]{2}{*}{$\mathrm{C}_{2} \mathrm{H}_{3} \mathrm{NH}_{2}-3 \mathrm{H}_{2} \mathrm{O}$} & 870 & 3.91 & $\omega_{\mathrm{NH}_{2}}$ & 40.29 \\
\hline & 838 & 5.92 & $\omega_{\mathrm{NH}_{2}}$ & \\
\hline \multirow[t]{2}{*}{$\mathrm{C}_{2} \mathrm{H}_{3} \mathrm{NH}_{2}-5 \mathrm{H}_{2} \mathrm{O}$} & 977 & 9.79 & $\omega_{\mathrm{NH}_{2}}$ & 41.30 \\
\hline & 838 & 7.77 & $\omega_{\mathrm{CH}_{2}}$ & \\
\hline $\mathrm{C}_{2} \mathrm{H}_{3} \mathrm{NH}_{2}-\mathrm{H}_{2} \mathrm{O}-\mathrm{SMD}$ & 761 & 10.79 & $\omega_{\mathrm{NH} 2}$ & 13.47 \\
\hline \multirow[t]{3}{*}{$\mathrm{C}_{2} \mathrm{H}_{3} \mathrm{NH}_{2}-3 \mathrm{H}_{2} \mathrm{O}-\mathrm{SMD}$} & 826 & 18.18 & $\omega_{\mathrm{NH}_{2}}$ & 19.66 \\
\hline & 812 & 37.56 & $\omega_{\mathrm{NH}_{2}}$ & \\
\hline & 772 & 9.14 & $\omega_{\mathrm{NH}_{2}}$ & \\
\hline \multirow[t]{2}{*}{$\mathrm{C}_{2} \mathrm{H}_{3} \mathrm{NH}_{2}-5 \mathrm{H}_{2} \mathrm{O}-\mathrm{SMD}$} & 820 & 50.03 & $\omega_{\mathrm{NH}_{2}}, \omega_{\mathrm{CH}_{2}}$ & 24.31 \\
\hline & 804 & 24.12 & $\omega_{\mathrm{NH}_{2}}, \omega_{\mathrm{CH}_{2}}$ & \\
\hline
\end{tabular}

$\omega_{\mathrm{NH}_{2}}$ : the $\mathrm{NH}_{2}$ wagging; $\omega_{\mathrm{CH}_{2}}$ : the $\mathrm{CH}_{2}$ wagging; $\gamma$ : out-of-plane

曲坐标与环呼吸振动坐标或 $\mathrm{C}-\mathrm{H}$ 面外弯曲坐标的 耦合. 随着水簇的增大, 三个谱峰的频率均有蓝移 的趋势. 对于乙烯胺与水分子作用, 如图 3(C) 所示, $\omega_{\mathrm{NH}_{2}}$ 的频率也随着水簇的增大发生显著蓝移. 在这 种情况下, 对应于 $\omega_{\mathrm{CH}_{2}}$ 的拉曼谱峰位于 $838 \mathrm{~cm}^{-1}$, 其 信号在频率和强度上几乎保持不变, 可见氨基与水 簇的作用对它的影响较小.

为了模拟水体相中分子拉曼光谱的变化, 采用 SMD 模型考虑溶剂化效应在氢键作用上的影响. 由 图 1 给出氢键结构参数可见, 考虑溶剂化后, 分子与 $\mathrm{H}_{2} \mathrm{O}$ 作用的 $\mathrm{N} \cdots \mathrm{H}-\mathrm{O}$ 氢键的距离仍随水分子数目 增加保持缩短趋势. ${ }^{10,11}$ 相对于气相情况, 在两个分 子与一个和三个水分子形成的簇中, 溶剂化效应导
致 $\mathrm{N} \cdots \mathrm{H}-\mathrm{O}$ 氢键的距离均缩短, 而当两个分子与五 个水分子形成的簇中, $\mathrm{N} \cdots \mathrm{H}-\mathrm{O}$ 氢键的距离略有增 长. 在考虑溶剂化条件下, 采用 $\mathrm{NBO}$ 分析计算了 $\mathrm{N} \cdots \mathrm{H}-\mathrm{O}$ 氢键的轨道作用能, 得到在苯胺复合物中 其分别为 $28.20 、 34.85$ 和 $41.84 \mathrm{~kJ} \cdot \mathrm{mol}^{-1}$, 而在乙烯胺 复合物中其依次为 $27.24 、 31.88$ 和 $38.79 \mathrm{~kJ} \cdot \mathrm{mol}^{-1}$. 与 气相 NBO 计算的轨道作用能相比, 前两者是增强 的, 后者是减小的. 这与我们计算的氢键距离的变 化相吻合, 表明虽然溶剂化作用导致贡献到氢键作 用的静电作用能减小, 但轨道作用相对增强, 并且 在三个复合物中趋向互相接近.

图 3(B) 和 3(D) 是在溶剂化下苯胺和乙烯胺与 水簇复合体系的计算拉曼光谱. 考虑溶剂化后, 计 

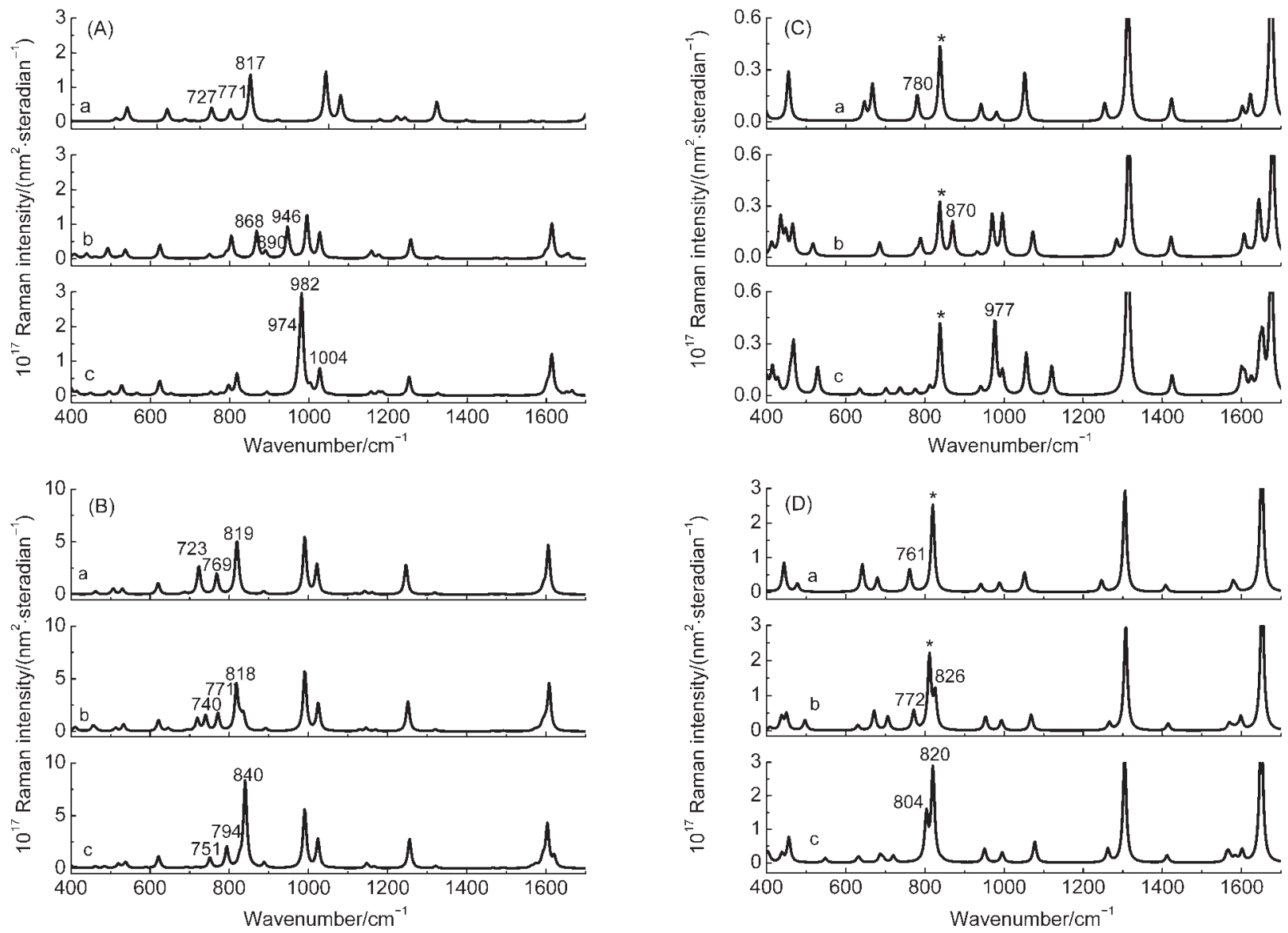

图 3 在 $\mathrm{B} 3 \mathrm{LYP} / 6-311+\mathrm{G}(d, p)$ 水平下计算的苯胺 $(\mathrm{A}, \mathrm{B})$ 和乙烯胺 $(\mathrm{C}, \mathrm{D})$ 与一个 $(\mathrm{a})$, 三个(b)和五个(c)水分子作用的拉曼光谱图

Fig.3 Simulated Raman spectra of aniline (A, B) and vinylamine (C, D) interacting with one (a), three (b), and five (c) water molecules calculated at the B3LYP/6-311+G(d,p) level

The incident wavelength of $514.5 \mathrm{~nm}$ was used here with Lorentzian line width of $10 \mathrm{~cm}^{-1}$. (A) and (C) are calculated in the gas phase;

(B) and (D) are calculated with the SMD model in aqueous solution. Peaks labeled with an asterisk belong to $\omega_{\mathrm{CH} 2}$.

算拉曼光谱显著不同于在气相条件下计算的拉曼 光谱. 虽然 $\omega_{\mathrm{NH}_{2}}$ 振动谱峰的频率蓝移减小, 谱峰强度 增加, 但计算得到拉曼谱的线形随水簇大小的变化 明显减小. 对于苯胺分子, 氢键作用使 $\omega_{\mathrm{NH}_{2}}$ 频率位移 至 818 或 $840 \mathrm{~cm}^{-1}$. 在 $500-1400 \mathrm{~cm}^{-1}$ 区间, 该拉曼谱 峰是较强的拉曼谱峰之一. 这与实验测得的苯胺液 体和晶体以及苯胺的饱和水溶液的拉曼光谱都非 常相似. ${ }^{39-42}$ 这是因为三者的氢键作用相近. ${ }^{43}$ 但前人 将该谱峰指认为苯胺的环呼吸振动 ${ }^{44}$ 或与氨基有关 的其它振动. ${ }^{41}$ 依据计算结果, 我们认为在苯胺液体 中分子间氢键可能导致其 $\omega_{\mathrm{NH}_{2}}$ 频率蓝移至 $820 \mathrm{~cm}^{-1}$, 其与苯胺环呼吸振动耦合. 在图 3(B)中, 高波数区 有三个相对较强的谱峰, 它们依次归属为苯环的三 角畸变、苯环上 $\mathrm{C}-\mathrm{H}$ 面内弯曲和 $\mathrm{C}-\mathrm{N}$ 伸缩振动. 它们的振动谱峰在频率和强度上均不随水分子数 目变化. 图3(D)是在考虑溶剂化后乙烯胺分子的计
算拉曼光谱. 在溶剂化后, 在不同的水簇中其 $\omega_{\mathrm{NH}_{2}}$ 频 率蓝移程度减小, 强度显著增加. 如表 2 所示, 在乙 烯胺与五个水分子作用时, 两种面外弯曲振动发生 耦合, 且在溶剂化条件下, 面外弯曲振动模受水簇 大小的影响很小, 谱图趋于一致.

上述结果表明, 无论苯胺还是乙烯胺, 与水簇 作用后, 氨基面外弯曲振动的频率与甲胺的非常接 近. 这说明分子之间的弱氢键作用导致苯胺和乙烯 胺中氨基的杂化性质与甲胺的接近. 为了进一步说 明这种振动模对弱相互作用的敏感性, 下面我们研 究了这两个分子与银簇的吸附作用.

\section{3 分子与银簇的作用对氨基面外弯曲振动模的 影响}

表 3 给出乙烯胺与四个不同的银簇作用的结构 参数和成键能. 乙烯胺与银簇作用的成键能在 $15-59 \mathrm{~kJ} \cdot \mathrm{mol}^{-1}$ 之间, 属于弱的化学吸附甚至物理 
表 $3 \mathrm{B3LYP/6-311G}(d, p) / \mathrm{LANL2DZ}(\mathrm{Ag})$ 水平下计算的

$\mathrm{C}_{2} \mathrm{H}_{3} \mathrm{NH}_{2}-\mathrm{Ag}_{n}(n=1,2,3,4)$ 复合物的结构参数和成键能

Table 3 Structural parameters and bonding energies (BE)

for $\mathrm{C}_{2} \mathrm{H}_{3} \mathrm{NH}_{2}-\mathrm{Ag}_{n}(n=1,2,3,4)$ complexes calculated at the B3LYP/6-311G $(d, p) / L A N L 2 D Z(A g)$ level

\begin{tabular}{cccccc}
\hline & Complex & $l_{\mathrm{C}-\mathrm{Ag}} / \mathrm{nm}$ & $l_{\mathrm{C}-\mathrm{C}} / \mathrm{nm}$ & $l_{\mathrm{N}-\mathrm{C}} / \mathrm{nm}$ & $\mathrm{BE} /\left(\mathrm{kJ} \cdot \mathrm{mol}^{-1}\right)$ \\
\hline (C-Ag) & $\mathrm{C}_{2} \mathrm{H}_{3} \mathrm{NH}_{2}-\mathrm{Ag}_{1}$ & 0.2699 & 0.1353 & 0.1374 & 14.98 \\
& $\mathrm{C}_{2} \mathrm{H}_{3} \mathrm{NH}_{2}-\mathrm{Ag}_{2}$ & 0.2501 & 0.1359 & 0.1366 & 37.15 \\
& $\mathrm{C}_{2} \mathrm{H}_{3} \mathrm{NH}_{2}-\mathrm{Ag}_{3}$ & 0.2437 & 0.1365 & 0.1363 & 48.16 \\
& $\mathrm{C}_{2} \mathrm{H}_{3} \mathrm{NH}_{2}-\mathrm{Ag}_{4}$ & 0.2424 & 0.1366 & 0.1365 & 58.87 \\
$(\mathrm{~N}-\mathrm{Ag})$ & $\mathrm{C}_{2} \mathrm{H}_{3} \mathrm{NH}_{2}-\mathrm{Ag}_{1}$ & 0.2728 & 0.1334 & 0.1406 & 12.68 \\
& $\mathrm{C}_{2} \mathrm{H}_{3} \mathrm{NH}_{2}-\mathrm{Ag}_{2}$ & 0.2462 & 0.1331 & 0.1420 & 34.94 \\
& $\mathrm{C}_{2} \mathrm{H}_{3} \mathrm{NH}_{2}-\mathrm{Ag}_{3}$ & 0.2414 & 0.1329 & 0.1424 & 41.92 \\
& $\mathrm{C}_{2} \mathrm{H}_{3} \mathrm{NH}_{2}-\mathrm{Ag}_{4}$ & 0.2395 & 0.1330 & 0.1424 & 52.22 \\
\hline
\end{tabular}

吸附. 无论是以氨基还是亚甲基与 $\mathrm{Ag}_{4}$ 作用时, 其成 键能都是最大的. $\mathrm{Ag}_{4}$ 簇的这种特殊成键作用主要 是它能提供合适的未占据轨道, 与氨基氮原子孤对 形成配位键. Zhao 等 ${ }^{18}$ 在研究苯胺与银簇的作用中 发现, 氨基氮上的孤对轨道与 $\mathrm{Ag}_{4}$ 簇的成键最强. Metiu 等 45,46 研究丙烯和 DNA 碱基与不同银簇作用 时, 也发现不同配体与 $\mathrm{Ag}_{4}$ 簇作用成键能最大, 复合 物体系更为稳定. 因此, 我们主要用 $\mathrm{Ag}_{4}$ 作为表面吸 附活性位来模拟分子以氨基吸附在银表面的情况. 如图 4所示, 从成键轨道的电子云图可以看出, 当乙 烯胺分别以亚甲基和氨基与 $\mathrm{Ag}_{4}$ 簇作用时, 分子是 以 $\pi$ 轨道或者氨基的孤对轨道与 $\mathrm{Ag}_{4}$ 簇的未成键 $s$ 轨 道作用的.

乙烯胺分子同时含有碳碳双键和氨基孤对电 子, 在自由分子中亚甲基和氨基的面外弯曲振动的 频率分别为 813 和 $544 \mathrm{~cm}^{-1}$. 它在银表面存在两种可 能吸附构型, 如图 4所示, (i) 分子以氨基上氮的孤对 轨道与银簇作用和(ii) 分子以 $\pi$ 轨道与银簇作用. 我 们的计算结果(如表 4 所示)表明当分子以氨基与银 簇作用时, 会使分子上氨基与碳碳双键之间的 $p-\pi$

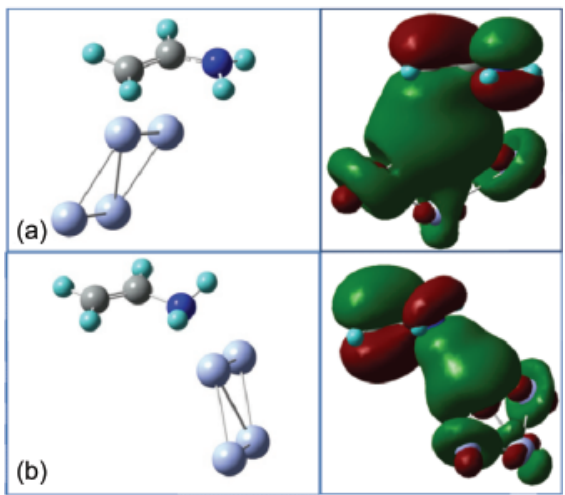

图 4 乙烯胺分子分别以亚甲基(a)和氨基(b)与 $\mathrm{Ag}_{4}$ 作用的 优化结构及成键轨道图

Fig.4 Optimized structures and the plots of bonding orbitals for vinylamine interacting with a silver cluster $\mathbf{A g}_{4}$ via methylene group (a) and amino group (b)

共轭效应减弱. 一方面, $\mathrm{C}-\mathrm{N}$ 键长增加, 电荷从氮 上转移到银簇加强了乙烯胺与银之间的相互作用, 此时氨基的翻转角从自由分子中的 $37.1^{\circ}$ 增大到 $48.5^{\circ}$, 氨基氮原子的 $s p^{3}$ 杂化性质增加. 这导致 $\omega_{\mathrm{NH}_{2}}$ 振动的频率升高到 $901 \mathrm{~cm}^{-1}$, 与自由分子中该振动 模的拉曼强度比较, 吸附态的拉曼强度增大约 15 倍. 另一方面, 这一作用也使 $\mathrm{C}=\mathrm{C}$ 键长显著缩短, 导致 $\pi$ 键的定域性质增强, 因此 $\omega_{\mathrm{CH}_{2}}$ 振动的频率发生 蓝移. 当分子以 $\pi$ 键与银簇作用时, $\mathrm{C}=\mathrm{C}$ 键长变长, 导致 $\omega_{\mathrm{CH} 2}$ 振动频率降低了 $5 \mathrm{~cm}^{-1}$. 此时靠近氨基碳 上的 $p$ 轨道倾向于与氨基的孤对轨道作用, 这使得 $\mathrm{C}-\mathrm{N}$ 键长缩短. 氨基的面外弯曲振动和摇摆振动 耦合并转变为两个 $\mathrm{NH}$ 的面外弯曲振动.

苯胺分子与银簇作用后, 氨基的翻转角由自由 分子的 $39.2^{\circ}$ 增大到 $50.6^{\circ}$, 此时氮原子上的孤对电 子与苯环的共轭效应减弱, 导致 $\mathrm{C}-\mathrm{N}$ 键长伸长, 而 氨基氮原子的 $s p^{3}$ 杂化性质增加, 因此 $\omega_{\mathrm{NH}_{2}}$ 的频率升 高到 $934 \mathrm{~cm}^{-1}$, 拉曼强度增大约 15 倍. 比较乙烯胺和

表 $4 \mathrm{B3LYP/6-311G}(d, p) / \mathrm{LANL2DZ}(\mathrm{Ag})$ 水平下计算乙烯胺和苯胺与 $\mathrm{Ag}_{4}$ 复合物的翻转角 $(\theta) 、 \mathrm{C}-\mathrm{C}$ 键长 $(l)$ 等结构参数、 面外弯曲振动模的频率 $\left(\omega_{i}\right)$ 和拉曼强度 $\left(S_{i}\right)$

Table 4 Inversion angle $(\theta)$, structural parameters $(l)$, vibrational frequencies $\left(\omega_{i}\right)$, and Raman intensities $\left(S_{i}\right)$ of wagging modes for $\mathrm{C}_{2} \mathrm{H}_{3} \mathrm{NH}_{2}, \mathrm{C}_{6} \mathrm{H}_{5} \mathrm{NH}_{2}$ and their complexes with $\mathrm{Ag}_{4}$ calculated at the B3LYP/6-311+G(d,p)/LANL2DZ level

\begin{tabular}{cccccc}
\hline Species & $\theta /\left(^{\circ}\right)$ & $l_{\mathrm{c}-\mathrm{c}} / \mathrm{nm}$ & $l_{\mathrm{C}(\mathrm{N})-\mathrm{Ag}} / \mathrm{nm}$ & $\omega_{i} / \mathrm{cm}^{-1}$ & $10^{4} S_{i} /\left(\mathrm{nm}^{4} \cdot \mathrm{amu}^{-1}\right)$ \\
\hline $\mathrm{C}_{2} \mathrm{H}_{3} \mathrm{NH}_{2}$ & 0 & 0.1334 & & $813\left(\mathrm{CH}_{2}\right)$ & 8.39 \\
& 37.1 & & & $544\left(\mathrm{NH}_{2}\right)$ & 3.86 \\
$\mathrm{C}_{2} \mathrm{H}_{3} \mathrm{NH}_{2}-\mathrm{Ag}_{4}(\mathrm{C}-\mathrm{Ag})$ & 16.1 & 0.1366 & 0.2424 & $808\left(\mathrm{CH}_{2}\right)$ & 64.87 \\
$\mathrm{C}_{2} \mathrm{H}_{3} \mathrm{NH}_{2}-\mathrm{Ag}_{4}(\mathrm{~N}-\mathrm{Ag})$ & 48.5 & 0.1330 & 0.2394 & $901\left(\mathrm{NH}_{2}\right)$ & 58.86 \\
& 0 & & & $874\left(\mathrm{CH}_{2}\right)$ & 2.12 \\
$\mathrm{C}_{6} \mathrm{H}_{5} \mathrm{NH}_{2}$ & 39.2 & 0.1398 & & 564 & 6.88 \\
$\mathrm{C}_{6} \mathrm{H}_{5} \mathrm{NH}_{2}-\mathrm{Ag}_{4}$ & 50.6 & 0.1433 & 0.2385 & 934 & 101.04 \\
\hline
\end{tabular}


苯胺分子以氨基与银簇作用, 发现二者的氨基面外 弯曲振动模的频率蓝移的程度和拉曼强度增加的 倍数非常接近. 同时, 结果表明弱相互作用对这种 $p-\pi$ 共轭体系的氨基面外弯曲振动的影响均较大.

\section{4 结 论}

通过密度泛函理论计算, 分析了苯胺和乙烯胺 与水簇的氢键作用以及它们和银簇的弱吸附作用. 我们发现这两类弱的相互作用显著影响分子内 $p-\pi$ 共轭效应, 从而导致分子内氨基面外弯曲振动的拉 曼光谱发生显著变化, 如在气相中, 弱的作用导致 拉曼谱峰发生显著蓝移. 分子以氨基与水簇或金属 簇作用后, 氨基氮的 $s p^{3}$ 杂化性质增强, 氨基的翻转 角增大导致 $p-\pi$ 共轭作用变化. 这种弱的作用也导 致氨基面外弯曲振动模的拉曼强度增大约 15 倍. 最 后, 理论研究结果也建议含这类氨基分子的拉曼信 号可能用作为一种分子探针, 通过分析拉曼信号, 研究分子与表面或环境分子的成键性质, 推断分子 的成键或吸附状态.

\section{References}

(1) Krueger, P. Canadian Journal of Chemistry 1963, 41, 363. doi: 10.1139/v63-053

(2) Xie, M., X.; Liu, Y. Spectrochimica Acta Part A: Molecular and Biomolecular Spectroscopy 2002, 58, 2817. doi: 10.1016/ S1386-1425(02)00072-0

(3) Solca, N.; Dopfer, O. The European Physical Journal D-Atomic, Molecular, Optical and Plasma Physics 2002, 20, 469.

(4) Szatylowicz, H.; Krygowski, T. M.; Hobza, P. The Journal of Physical Chemistry A 2007, 111, 170. doi: 10.1021/jp065336v

(5) Wolff, H.; Mathias, D. The Journal of Physical Chemistry 1973, 77, 2081. doi: 10.1021/j100636a010

(6) Nakanaga, T.; Ito, F.; Miyawaki, J.; Sugawara, K.; Takeo, H. Chemical Physics Letters 1996, 261, 414. doi: 10.1016/ 0009-2614(96)00994-3

(7) Nakanaga, T.; Kawamata, K.; Ito, F. Chemical Physics Letters 1997, 279, 309. doi: 10.1016/S0009-2614(97)01059-2

(8) Nakanaga, T.; Sugawara, K.; Kawamata, K.; Ito, F. Chemical Physics Letters 1997, 267, 491. doi: 10.1016/S0009-2614(97) 00127-9

(9) Schmid, R.; Chowdhury, P.; Miyawaki, J.; Ito, F.; Sugawara, K.; Nakanaga, T.; Takeo, H.; Jones, H. Chemical Physics 1997, 218 , 291. doi: 10.1016/S0301-0104(97)00072-4

(10) Fedotova, M. V.; Kruchinin, S. E. Journal of Molecular Liquids 2013, 179, 27. doi: 10.1016/j.molliq.2012.11.031

(11) Plugatyr, A.; Svishchev, I. M. The Journal of Chemical Physics 2009, 130, 114509. doi: 10.1063/1.3096672
(12) Li, D. W.; Qu, L. L.; Zhai, W. L.; Xue, J. Q.; Fossey, J. S.; Long, Y. T. Environmental Science \& Technology 2011, 45, 4046. doi: $10.1021 / \mathrm{es} 104155 \mathrm{r}$

(13) Perry, D. A.; Cordova, J. S.; Schiefer, E. M.; Chen, T. Y.; Razer, T. M.; Biris, A. S. The Journal of Physical Chemistry C 2012, 116, 4584. doi: 10.1021/jp208489w

(14) Wu, D. Y.; Liu, X. M.; Huang, Y. F.; Ren, B.; Xu, X.; Tian, Z. Q. The Journal of Physical Chemistry C 2009, 113, 18212. doi: 10.1021/jp9050929

(15) Tian, Z. Q.; Lei, L. C.; Jing, X. B. Acta Phys. -Chim. Sin. 1988, 4, 458. [田中群, 雷良才, 景遐斌. 物理化学学报, 1988, 4, 458.] doi: 10.3866/PKU.WHXB19880504

(16) Park, H.; Lee, S. B.; Kim, K.; Kim, M. S. The Journal of Physical Chemistry 1990, 94, 7576.

(17) Park, S. H.; Kim, K.; Kim, M. S. Journal of Molecular Structure 1993, 301, 57. doi: 10.1016/0022-2860(93)80231-J

(18) Zhao, L. B.; Huang, R.; Bai, M. X.; Wu, D. Y.; Tian, Z. Q. The Journal of Physical Chemistry C 2011, 115, 4174. doi: 10.1021/ jp1117135

(19) Hamada, Y.; Sato, N.; Tsuboi, M. Journal of Molecular Spectroscopy 1987, 124, 172. doi: 10.1016/0022-2852(87) 90130-5

(20) McNaughton, D.; Evans, C. J. Journal of Molecular Spectroscopy 1999, 196, 274. doi: 10.1006/jmsp.1999.7861

(21) Brown, R.; Godfrey, P.; Kleibomer, B.; Pierlot, A.; McNaughton, D. Journal of Molecular Spectroscopy 1990, 142, 195. doi: 10.1016/0022-2852(90)90177-R

(22) Marenich, A. V.; Cramer, C. J.; Truhlar, D. G. The Journal of Physical Chemistry B 2009, 113, 6378. doi: 10.1021/jp810292n

(23) Wu, D. Y.; Hayashi, M.; Shiu, Y. J.; Liang, K. K.; Chang, C. H.; Yeh, Y. L.; Lin, S. H. The Journal of Physical Chemistry A 2003, 107, 9658. doi: 10.1021/jp0349511

(24) Frisch, M. J.; Trucks, G. W.; Schlegel, H. B.; et al. Gaussian 09, Revision A. 01; Gaussian Inc.: Wallingford, CT, 2009.

(25) McLean, A.; Chandler, G. The Journal of Chemical Physics 1980, 72, 5639. doi: 10.1063/1.438980

(26) Krishnan, R.; Binkley, J. S.; Seeger, R.; Pople, J. A. The Journal of Chemical Physics 1980, 72, 650. doi: 10.1063/1.438955

(27) Wadt, W. R.; Hay, P. J. The Journal of Chemical Physics 1985, 82, 284. doi: $10.1063 / 1.448800$

(28) Hay, P. J.; Wadt, W. R. The Journal of Chemical Physics 1985, 82, 299. doi: 10.1063/1.448975

(29) Schultz, G.; Portalone, G.; Ramondo, F.; Domenicano, A.; Hargittai, I. Structural Chemistry 1996, 7, 59. doi: 10.1007/ BF02275450

(30) Boys, S.; Bernardi, F. Molecular Physics 1970, 19, 553. doi: 10.1080/00268977000101561

(31) Reed, A. E.; Curtiss, L. A.; Weinhold, F. Chemical Reviews 1988, 88, 899. doi: 10.1021/cr00088a005

(32) Carpenter, J.; Weinhold, F. Journal of Molecular Structure: Theochem 1988, 169, 41. doi: 10.1016/0166-1280(88)80248-3 
(33) Liang, X. J.; Cui, L.; Wu, D. Y.; Tian, Z. Q. Acta Phys. -Chim. Sin. 2009, 25, 1605 [梁晓静, 崔 丽, 吴德印, 田中群. 物理 化学学报, 2009, 25, 1605.] doi: 10.3866/PKU.WHXB20090808

(34) Ziegler, L.; Hudson, B. The Journal of Physical Chemistry 1984, 88 , 1110. doi: 10.1021/j150650a016

(35) Tsuboi, M.; Hirakawa, A. Y.; Ino, T.; Sasaki, T.; Tamagake, K The Journal of Chemical Physics 1964, 41, 2721. doi: 10.1063/ 1.1726344

(36) Evans, J. Spectrochimica Acta 1960, 16, 428. doi: 10.1016/ 0371-1951(60)80037-9

(37) Larsen, N.; Hansen, E.; Nicolaisen, F. Chemical Physics Letters 1976, 43, 584. doi: 10.1016/0009-2614(76)80629-X

(38) Rauhut, G.; Pulay, P. The Journal of Physical Chemistry 1995, 99, 3093. doi: 10.1021/j100010a019

(39) Shindo, H. J. Chem. Soc. Faraday Trans. 1 1986, 82, 45. doi: $10.1039 / \mathrm{f} 19868200045$
(40) Holze, R. Journal of Electroanalytical Chemistry and Interfacial Electrochemistry 1988, 250, 143. doi: 10.1016/ 0022-0728(88)80199-2

(41) Tripathi, G. The Journal of Chemical Physics 1980, 73, 5521. doi: $10.1063 / 1.440072$

(42) Qi, Y.; Hu, Y.; Xie, M.; Xing, D.; Gu, H. Journal of Raman Spectroscopy 2011, 42, 1287. doi: 10.1002/jrs.v42.6

(43) Funnell, N. P.; Dawson, A.; Marshall, W. G.; Parsons, S. CrystEngComm 2013, 15, 1047.

(44) Wojciechowski, P. M.; Zierkiewicz, W.; Michalska, D.; Hobza, P. The Journal of Chemical Physics 2003, 118, 10900. doi: $10.1063 / 1.1574788$

(45) Chrétien, S.; Gordon, M. S.; Metiu, H. The Journal of Chemical Physics 2004, 121, 9925. doi: 10.1063/1.1809600

(46) Soto-Verdugo, V.; Metiu, H.; Gwinn, E. The Journal of Chemical Physics 2010, 132, 195102. doi: 10.1063/1.3419930 